\title{
Architecture of hydrogen-bonded anionic substructure vs cation type in thiazolium hypodiphosphates
}

\author{
D. Budzikur, K. Ślepokura \\ University of Wrockaw, Faculty of Chemistry, 14. F. Joliot-Curie, 50-383 Wroctaw \\ daria.budzikur@chem.uni.wroc.pl
}

Hypodiphosphoric acid $\left(\mathrm{H}_{4} \mathrm{P}_{2} \mathrm{O}_{6}\right)$ - the structural analogue of diphosphoric acid $\left(\mathrm{H}_{4} \mathrm{P}_{2} \mathrm{O}_{7}\right)$ - contains two phosphorus atoms at the +4 oxidation state, which are connected by a direct covalent bond. After its synthesis was described in the 19th century, the mainstream of scientific research focused on the synthesis and physicochemical properties of its inorganic salts $[1,2]$.

In recent years, research has been intensified on organic-inorganic hybrids, including organic hypodiphosphates [3-5]. The lack of an oxygen bridge in hypodiphosphates contributes to their slightly higher stability (compared to diphosphates) and makes the ions/molecules more rigid. At the same time they still have six oxygen atoms capable of participating in the formation of networks stabilized by strong $\mathrm{O}-\mathrm{H} \cdots \mathrm{O}$ hydrogen bonds, composed exclusively of hypodiphosphate anions and/or acid molecules. This is confirmed by the known hypodiphosphate crystals characterized by the presence of such substructures, with different architectures and dimensions.

In the present poster, the effect of methyl or amino substitution of thiazole ring on the hydrogen bond patterns observed in eight thiazolium hypodiphosphate crystals will be analyzed. It was found that the presence of one-, two- or three-dimensional anionic substructures was related to the degree of anion ionization and the size of the cation used. Monoanions form 3D hypodiphosphate networks, while ionic cocrystals containing dianions and hypodiphosphoric acid molecules (depending on the cation type) - 1D or 2D substructures. The presence of water molecules in the crystal results in the formation of higher dimensional inorganic substructures.

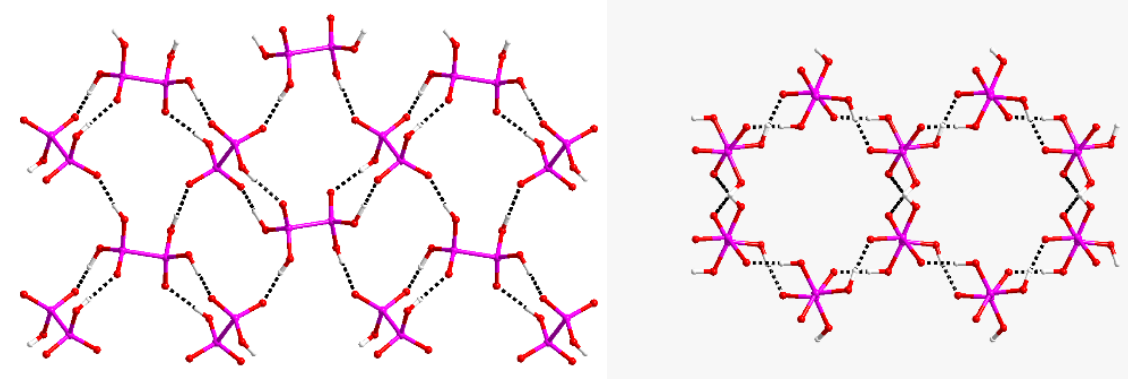

Figure 1. Exemplary multidimensional hydrogen-bonded anionic substructures observed in thiazolium hypodiphosphate and its derivatives.

[1] Salzer, T. (1987). Liebigs Ann. 187, 322.

[2] Kinzhybalo, V., Otręba, M., Ślepokura, K. \& Lis, T. (2021). Wiad. Chem. 75, 423.

[3] Otręba, M., Budzikur, D., Górecki, Ł. \& Ślepokura. K. (2018). Acta Cryst. C74, 571.

[4] Emami, M., Ślepokura, K. A., Trzebiatowska, M., Noshiranzadeh, N. \& Kinzhybalo, V. (2018). CrystEngComm.. $20,5207$.

[5] Budzikur, D., Szklarz, P., Kinzhybalo, V. \& Ślepokura. K. (2020). Acta Cryst. B76, 939.

Keywords: Hypodiphosphate, organic-inorganic hybrid, crystal structure, anionic network, thiazolium salts 